\title{
Papers
}

\section{Population based randomised controlled trial on impact of screening on mortality from abdominal aortic aneurysm}

Paul E Norman, Konrad Jamrozik, Michael M Lawrence-Brown, Max T Q Le, Carole A Spencer, Raywin J Tuohy, Richard W Parsons, James A Dickinson

\begin{abstract}
Objective To assess whether screening for abdominal aortic aneurysms in men reduces mortality.

Design Population based randomised controlled trial of ultrasound screening, with intention to treat analysis of age standardised mortality.

Setting Community based screening programme in Western Australia.

Participants 41000 men aged 65-83 years randomised to intervention and control groups.

Intervention Invitation to ultrasound screening.

Main outcome measure Deaths from abdominal aortic aneurysm in the five years after the start of screening.

Results The corrected response to invitation to screening was $70 \%$. The crude prevalence was $7.2 \%$ for aortic diameter $\geq 30$ $\mathrm{mm}$ and $0.5 \%$ for diameter $\geq 55 \mathrm{~mm}$. Twice as many men in the intervention group than in the control group underwent elective surgery for abdominal aortic aneurysm (107 v 54, $\mathrm{P}=0.002, \chi^{2}$ test). Between scheduled screening and the end of follow up 18 men in the intervention group and 25 in the control group died from abdominal aortic aneurysm, yielding a mortality ratio of 0.61 (95\% confidence interval 0.33 to 1.11 ). Any benefit was almost entirely in men aged between 65 and 75 years, where the ratio was reduced to 0.19 (0.04 to 0.89 ).

Conclusions At a whole population level screening for abdominal aortic aneurysms was not effective in men aged 65-83 years and did not reduce overall death rates. The success of screening depends on choice of target age group and the exclusion of ineligible men. It is also important to assess the current rate of elective surgery for abdominal aortic aneurysm as in some communities this may already approach a level that reduces the potential benefit of population based screening.
\end{abstract}

\section{Introduction}

Despite advances in vascular surgery and intensive care, the overall case fatality of rupture of an abdominal aortic aneurysm is still around $80 \% .{ }^{1}$ In addition, the incidence of such events seems to have risen over the past two decades. ${ }^{2-4}$ This contrasts with trends seen in coronary heart disease and stroke, suggesting that measures introduced to reduce the impact of occlusive arterial disease have not been effective.

The feasibility of population based screening for such aneurysms by using ultrasound has been established over the past 15 years. ${ }^{5-7}$ These early studies generated considerable debate about the merits of population screening, resulting in calls for randomised controlled trials. ${ }^{8-10}$ In the first such trial, Scott et al reported a large (50\%) but non-significant reduction in mortality with screening. ${ }^{11}$ Since then two randomised trials and several non-randomised studies have all indicated that screening in men saves lives cost effectively. ${ }^{12-16}$ We designed a trial to complement these, focusing on men aged 65 to 74 years but also including an older group.

The Western Australia trial of screening for abdominal aortic aneurysms is a population based randomised controlled trial to determine whether a single ultrasound screen for abdominal aortic aneurysms ${ }^{17}$ reduces deaths among those to whom it is offered. The trial was designed to cover an entire metropolitan community and assess the effectiveness of introducing population screening into an existing healthcare system. Western Australia is an ideal location for such a trial: the population is geographically isolated and stable, all deaths and hospital admissions are linked within a unified database system, and much is already known about local trends and outcomes for abdominal aortic aneurysm. ${ }^{13} 1819$ Here we report on the early effect of screening on mortality in the trial.

\section{Methods}

\section{Study population}

To provide the most rigorous possible test of the utility of screening, we designed the trial as a population based study with the primary end point, mortality from abdominal aortic aneurysm, to be analysed on an intention to treat basis. We planned to have $90 \%$ power to detect and declare significant (two sided $\alpha=0.05)$ a relative reduction of $50 \%$ in mortality among men invited for screening over five years from the start of screening. Using available pilot data, we estimated that the control group would need to contain about 20000 men to experience 55 deaths from abdominal aortic aneurysm. ${ }^{3}$

Men were identified from an electronic copy of the electoral roll, enrolment to vote being compulsory for all Australian adults. The ideal target age range for screening is $65-74$ years, but the electoral roll listed fewer than 40000 men in this age group in the main metropolitan area of Perth (the capital city of Western Australia) and satellite towns. The addition of men aged 75-79 years increased the potentially available number to about 50000 . We excluded 8801 because they lived in the furthest satellite town (about $35 \mathrm{~km}$ away from Perth), and we did not have the resources to set up a screening clinic in this location. We achieved our required sample size without including them.

At the beginning of the trial we selected all 41000 men on the electoral roll who were resident in Perth and were expected to be 65-79 years old at the projected mid-point of screening. 


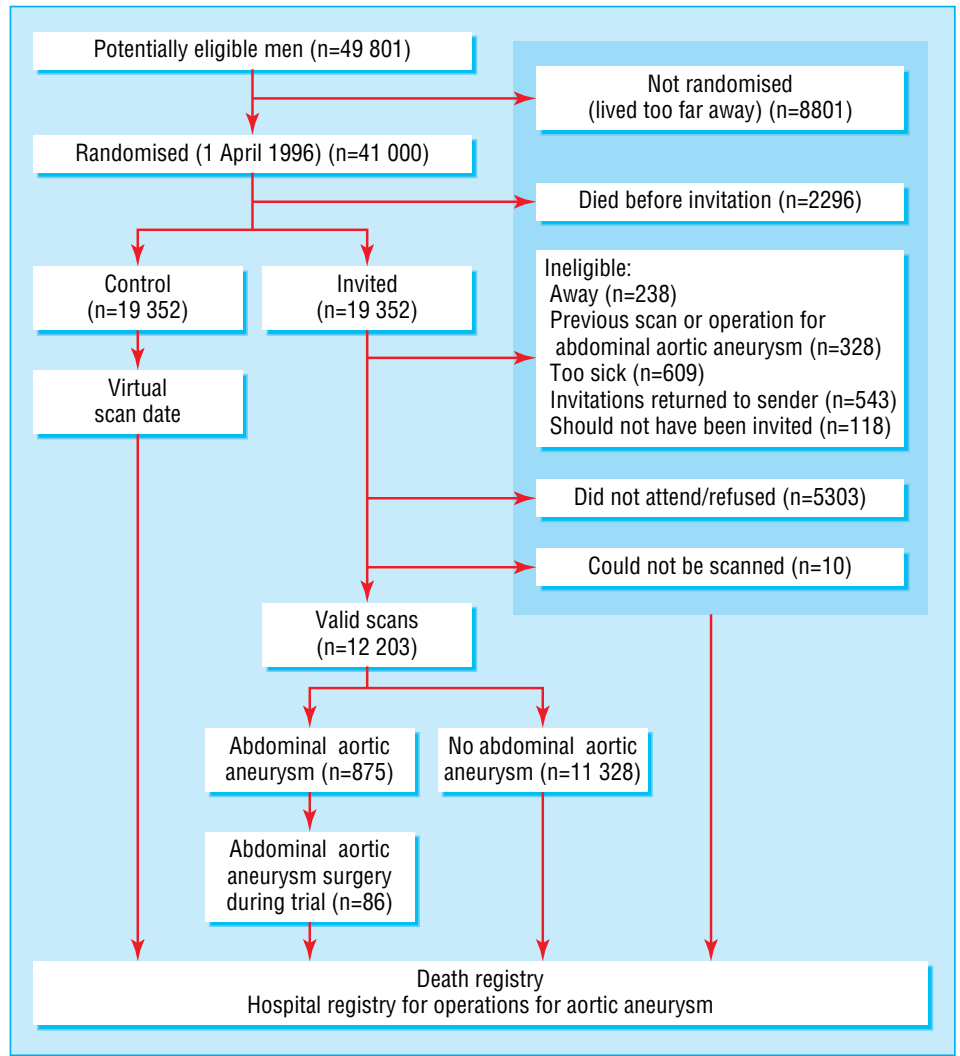

Fig 1 Summary of trial of screening for abdominal aortic aneurysm

Men were randomised into intervention and control groups of equal size defined by five year age group and postcode. Men in the control group were allocated a "virtual" date of screening, which was the median scheduled date of examination for men from the same postcode area randomised to the intervention group. Men in the intervention group were sent a letter explaining the study and an appointment for a scan. Men who did not take up the initial invitation to screening were sent a second letter.

At the five screening clinics the greatest transverse and anteroposterior diameter was measured with a Toshiba Capasee ultrasound machine with a $3.75 \mathrm{MHz}$ probe (Toshiba Australia, North Ryde, NSW). On leaving the clinic, each man was given a letter containing the results of his scan, with a copy for his general practitioner. The general practitioner arranged any follow up investigations or referral to a surgeon. We made no attempt to influence any aspects of clinical management, in particular with regards to threshold for intervention or method of repair.

\section{Procedures for follow up}

We used electronic record linkage to population based named identified records for deaths and admissions to hospital in Western Australia to identify end points in the target population. When linkage to a death registration was confirmed, a researcher who was unaware of group allocation coded up to four causes of death from the text of the original death certificate. Deaths with mention of abdominal aortic aneurysm were identified for further blinded independent confirmation of the contribution to death of the aneurysm or surgery for the aneurysm. We also used electronic record linkage to identify all men undergoing surgery for abdominal aortic aneurysm, either before or after scheduled screening. For admission dates before 1988 we used a combination of international classification of disease, ninth revision,
(ICD-9) diagnosis codes (441.30 or 441.40) and International Classification for Procedures in Medicine (ICPM) code (58.53). Patients admitted from 1988 to 31 June 1999 were identified with the combined ICD-9 clinical modification (ICD-9-CM) diagnosis codes (441.30 or 441.40) and ICD-9-CM procedure code (38.44). Patients admitted after 1 July 1999 were identified with ICD-10 codes (I71.3 or I71.4).

\section{Statistical methods}

We initially compared crude and age standardised mortality from definite abdominal aortic aneurysm in the two groups between each man's actual or virtual date of screening and the end of follow up on 31 March 2001, which was five years after the trial began. Principal results are presented as ratios and corresponding 95\% confidence intervals for mortality for abdominal aortic aneurysm adjusted in single year strata to the age structure of the male population of Western Australia. To facilitate comparison with studies such as the multicentre aneurysm screening study (MASS), ${ }^{15}$ our secondary analyses included the mortality rate ratio for deaths from abdominal aortic aneurysm in men aged 64-75 years. Finally, we examined mortality from all causes over the five years of the programme.

\section{Results}

Figure 1 shows the flow of participants through the study. The 41000 men randomised to the two groups were balanced for age (mean (SD) 72.6 (4.7) v 72.6 (4.7) years). Screening was completed over a period of 32 months, and the median follow up was 43 (range 27-61) months. Because of the method of recording age in the electoral roll, some men were older than the target age range by the time they were invited for screening and, as a result, $725(5.9 \%)$ of those who attended were aged $80-83$ years. ${ }^{17}$ 
Table 1 Elective and emergency procedures for abdominal aortic aneurysm before scheduled screening

\begin{tabular}{lccc} 
Group & Elective & Emergency & Total $(\%)$ \\
\hline Scanned $(\mathrm{n}=12$ 203) & 20 & 2 & $22(0.2)$ \\
\hline Not scanned $(\mathrm{n}=8$ 297) & 198 & 29 & $227(2.7)$ \\
\hline Total invited $(\mathrm{n}=19352)$ & 218 & 31 & $249(1.3)$ \\
\hline Control $(\mathrm{n}=19$ 352) & 191 & 33 & $224(1.2)$
\end{tabular}

*Includes emergency symptomatic cases.

\section{Participation in screening and prevalence of abdominal aortic aneurysm}

There was no difference between the numbers of men in the two groups who had had surgery for abdominal aortic aneurysm before scheduled screening (table $1, \mathrm{P}=0.47, \chi^{2}$ test). Within the men invited for screening, the prevalence of previous surgery for abdominal aortic aneurysm was highest among those who did not attend (2.7\%). Similar numbers of men in the two groups died between randomisation and scheduled screening, including 25 deaths from rupture (13 in invited group, 12 in control group). After correcting the lists for deaths, we sent letters of invitation to 19352 men. Of these, 12213 underwent a screening examination, a crude response fraction of $63.1 \%$. If we excluded 1836 men who would generally be considered ineligible this figure increased to $70 \%$ (fig 1).

The overall crude prevalence of any aortic aneurysms (aorta $\geq 30 \mathrm{~mm}$ ) was $7.2 \%$, increasing from $4.8 \%$ in men aged $65-69$ years to $10.8 \%$ in the oldest men. Of the 875 cases detected, 699 $(80 \%)$ aortas were $30-44 \mathrm{~mm}$ in diameter, $115(13 \%)$ were $45-54$ $\mathrm{mm}$ in diameter, and $61(7 \%)$ were $\geq 55 \mathrm{~mm}$ in diameter. ${ }^{17}$

\section{Procedures and deaths after scheduled screening}

Table 2 shows the numbers of procedures for and deaths from abdominal aortic aneurysm after scheduled screening. Table 3 shows the corresponding numbers of events that occurred between randomisation and the end of follow up. The overall mortality within 30 days was 4.3\% (7/161) after elective surgery and 24\% (4/17) after surgery for ruptured aneurysms (table 2), with no difference between study groups $\left(\mathrm{P}=0.59, \chi^{2}\right.$ test). Twice

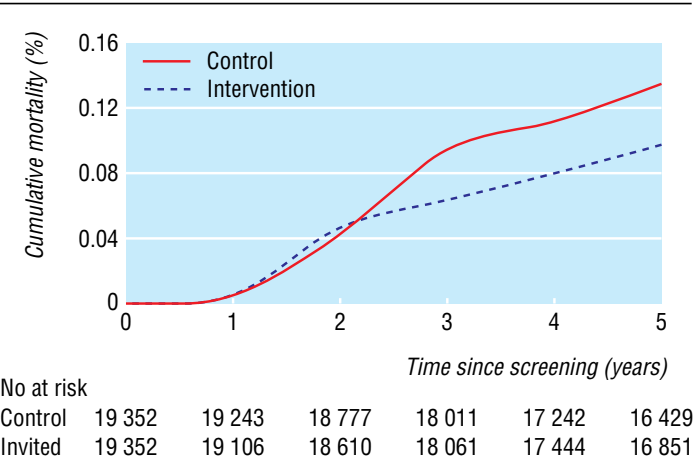

Fig 2 Cumulative mortality due to abdominal aortic aneurysm after date of screening

as many men in the intervention group underwent elective surgery for abdominal aortic aneurysm compared with the number in the control group (107 $v 54, \mathrm{P}=0.002, \chi^{2}$ test).

Between scheduled screening and the end of follow up 18 men died from abdominal aortic aneurysm in the intervention group and 25 in the control group (table 2), yielding a mortality rate ratio of 0.61 (95\% confidence interval 0.33 to 1.11$)$. The age standardised mortality for those who actually attended screening was $60 \%$ lower than in the control group (7.48 $v 18.91$ deaths per 100000 man years, table 2). Between randomisation and the end of follow up there were 31 deaths from abdominal aortic aneurysm in the intervention group and 37 in the control group, yielding a mortality rate ratio of 0.85 ( 0.53 to 1.36 , table 3 ). Figures 2 and 3 show the cumulative mortality from abdominal aortic aneurysm. Men in the intervention group initially had a higher mortality, but after one year the mortality curves crossed, such that the difference between them was not significant.

Table 4 summarises the mortality from abdominal aortic aneurysm in men aged $65-74$ years and $\geq 75$ years. There were no deaths among men aged 65-74 years who underwent screening and only two deaths (after scheduled screening) among those who were invited but did not attend compared with 10 in the

Table 2 Elective and emergency procedures and crude and age standardised mortality from abdominal aortic aneurysm between scheduled screening and the end of follow up

\begin{tabular}{|c|c|c|c|c|c|c|c|c|}
\hline & \multicolumn{2}{|c|}{ Elective } & \multicolumn{4}{|c|}{ Emergency } & \multirow[b]{2}{*}{ Total deaths (\%) } & \multirow[b]{2}{*}{$\begin{array}{l}\text { Age standardised } \\
\text { mortality* }(95 \% \text { CI) }\end{array}$} \\
\hline & Operation & $\begin{array}{l}\text { Postoperative } \\
\text { death }(\%)\end{array}$ & All ruptures & Operation & $\begin{array}{l}\text { Postoperative } \\
\text { death }\end{array}$ & $\begin{array}{c}\text { Fatal rupture without } \\
\text { surgery }\end{array}$ & & \\
\hline \multicolumn{9}{|l|}{ Intervention group } \\
\hline Scanned $(n=12203)$ & 86 & $4(4.7)$ & 3 & 0 & 0 & 3 & $7(0.06)$ & 7.48 (1.91 to 13.05$)$ \\
\hline Not scanned $(n=7$ 149) & 21 & 0 & 30 & 9 & 1 & 10 & $11(0.15)$ & 18.27 (7.08 to 29.46$)$ \\
\hline Total invited ( $\mathrm{n}=19352)$ & 107 & $4(3.7)$ & 33 & 9 & 1 & 13 & $18(0.09)$ & 11.51 (6.16 to 16.86) \\
\hline \multicolumn{9}{|l|}{ Control group } \\
\hline Total $(n=19352)$ & 54 & $3(5.6)$ & 38 & 8 & 3 & 19 & $25(0.13)$ & 18.91 (10.97 to 26.85 ) \\
\hline
\end{tabular}

*Age standardised per 100000 in strata of one year to age structure of Western Australian male population aged 65-83 years.

Table 3 Elective and emergency procedures, deaths, and crude and age standardised mortality from abdominal aortic aneurysm between randomisation and the end of follow up

\begin{tabular}{|c|c|c|c|c|c|c|c|c|}
\hline & \multicolumn{2}{|c|}{ Elective } & \multicolumn{4}{|c|}{ Emergency } & \multirow[b]{2}{*}{ Total deaths (\%) } & \multirow[b]{2}{*}{$\begin{array}{l}\text { Age standardised } \\
\text { mortality* }(95 \% \mathrm{Cl})\end{array}$} \\
\hline & Operation & $\begin{array}{l}\text { Postoperative } \\
\text { death }(\%)\end{array}$ & All ruptures & Operation & $\begin{array}{c}\text { Postoperative } \\
\text { death }\end{array}$ & $\begin{array}{l}\text { Fatal rupture without } \\
\text { surgery }\end{array}$ & & \\
\hline \multicolumn{9}{|l|}{ Intervention group } \\
\hline Scanned $(n=12203)$ & 86 & $4(4.7)$ & 3 & 0 & 0 & 3 & $7(0.06)$ & 7.48 (1.91 to 13.05$)$ \\
\hline Not scanned $(\mathrm{n}=8$ 297) & 26 & $1(3.9)$ & 35 & 11 & 2 & 21 & $24(0.29)$ & 46.56 (24.7 to 68.4) \\
\hline Total invited $(\mathrm{n}=20500)$ & 112 & $5(4.5)$ & 38 & 11 & 2 & 24 & $31 \quad(0.15)$ & 23.55 (13.79 to 33.31) \\
\hline \multicolumn{9}{|l|}{ Control group } \\
\hline Total $(n=20500)$ & 60 & $4(6.7)$ & 41 & 10 & 5 & 28 & $37(0.18)$ & 27.83 (16.89 to 38.77$)$ \\
\hline
\end{tabular}

*Age standardised per 100000 in strata of one year to age structure of Western Australian male population aged 65-83 years. 


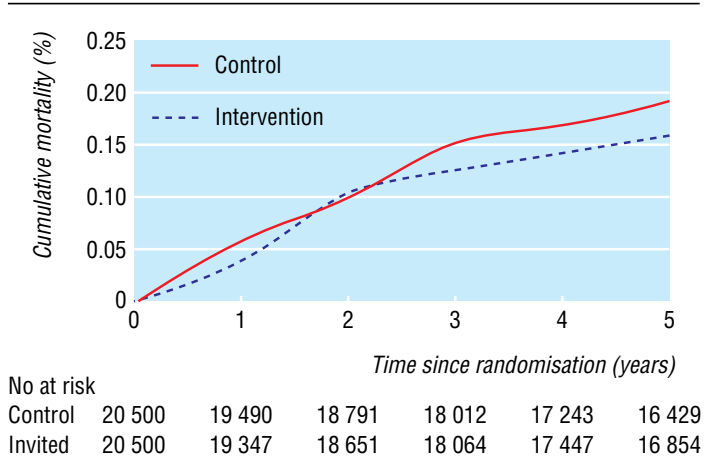

Fig 3 Cumulative mortality due to abdominal aortic aneurysm after date of randomisation

control group, an odds ratio of $0.19(0.04$ to $0.89, \mathrm{P}=0.01)$. When we included deaths between randomisation and scheduled screening, however, we found no benefit in the younger age group (odds ratio $0.82,0.37$ to $1.84, \mathrm{P}=0.6$ ).

Table 5 and figures 4 and 5 show the cumulative and age standardised all cause mortality. There is clear evidence of response bias among men who took up the invitation to be screened; their mortality experience was close to half that of invited men who did not undergo a scan. Overall, however, there were no meaningful differences in the age standardised mortality rates for all causes for the invited and control groups.

\section{Discussion}

The main conclusion from our population based randomised controlled trial of ultrasound screening for abdominal aortic aneurysm in Western Australia is that associated mortality for men aged 65-83 years is not significantly reduced by a screening programme. Although there was substantial benefit for the men who attended for screening, the overall benefit was reduced by

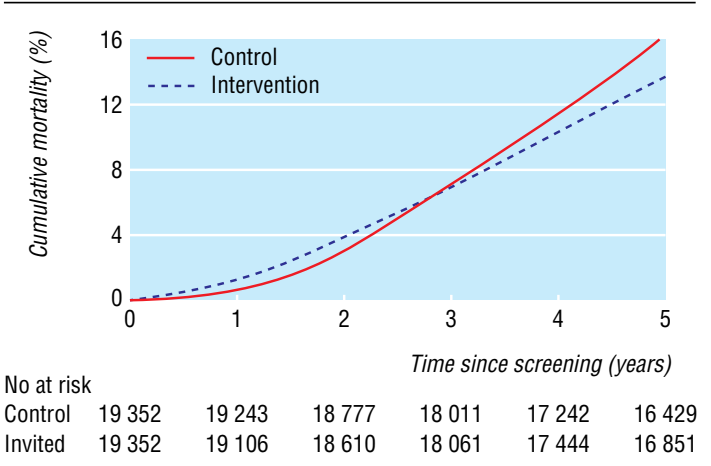

Fig 4 Cumulative all cause mortality after date of screening

deaths among men who did not attend. The multicentre aneurysm screening study (MASS) has already provided convincing evidence that screening men aged 65-74 years has the potential to reduce the mortality. ${ }^{15}$ In light of these findings, a target group aged 65-74 years may have been more suitable for our study. Indeed, subgroup analysis indicated that this group benefited the most from screening. For several reasons, however, we had to include 13688 (33.4\%) men aged 75-83 years. These men cannot be excluded post hoc and our primary intention to treat analysis included all randomised men aged 65-83 years.

\section{Method of recruitment}

In addition to the differences in target age range, our trial and the multicentre study used quite different methods of recruitment (electoral roll and general practice lists). As systematic screening is often based on centralised population databases rather than general practice lists, our trial is probably more representative of what would occur in many countries if screening were to be introduced. Because we used a centralised database, however, we could not exclude various categories of "ineligible" men before randomisation (fig 1). Thus we included men who

Table 4 Cumulative mortality from abdominal aortic aneurysm in men aged 64-75 years and $\geq 75$ years

\begin{tabular}{|c|c|c|c|c|}
\hline & \multicolumn{2}{|c|}{$65-74$ years } & \multicolumn{2}{|c|}{$\geq 75$ years } \\
\hline & No of men (person years) & Deaths & No of men (person years) & Deaths \\
\hline \multicolumn{5}{|l|}{ From randomisation } \\
\hline \multicolumn{5}{|l|}{ Intervention group: } \\
\hline Scanned & 8641 (30 462) & 0 & 3562 (13 011) & 7 \\
\hline Not scanned & $5200(17876)$ & 11 & 3097 (10 746) & 13 \\
\hline Total invited & 13841 (48 338) & 11 & $6659(23757)$ & 20 \\
\hline Control group & 13464 (46 912) & 13 & $7036(25046)$ & 24 \\
\hline Odds ratio $(95 \% \mathrm{Cl})$ & \multicolumn{2}{|c|}{0.82 (0.37 to 1.84$)$} & \multicolumn{2}{|c|}{0.88 (0.49 to 1.6$)$} \\
\hline \multicolumn{5}{|c|}{ From scheduled screening } \\
\hline \multicolumn{5}{|l|}{ Intervention group: } \\
\hline Scanned & 8641 (30 462) & 0 & 3562 (13 011) & 7 \\
\hline Not scanned & 4657 (16 104) & 2 & 2492 (8 743) & 9 \\
\hline Total invited & $13298(46566)$ & 2 & 6054 (21 754) & 16 \\
\hline Control group & 12938 (45 177) & 10 & 6414 (22 949) & 15 \\
\hline Odds ratio $(95 \% \mathrm{Cl})$ & \multicolumn{2}{|c|}{0.19 (0.04 to 0.89$)$} & \multicolumn{2}{|c|}{1.13 (0.56 to 2.29 ) } \\
\hline
\end{tabular}

Table 5 Cumulative and age standardised all cause mortality over five years after real or virtual date of screening

\begin{tabular}{|c|c|c|c|c|}
\hline Subgroup of men & No of men & Person years & Cumulative deaths $(\%)$ & Age standardised mortality ${ }^{*}(95 \% \mathrm{Cl})$ \\
\hline Scanned & 12203 & 43473 & $1071(8.8)$ & 1501 (1377 to 1625$)$ \\
\hline Not scanned & 7149 & 24847 & $1161(16.2)$ & 2885 (2691 to 3079 ) \\
\hline Total invited & 19352 & 68320 & $2232(11.5)$ & 1976 (1945 to 2117) \\
\hline Control & 19352 & 68126 & $2571(13.3)$ & 2020 (1948 to 2092) \\
\hline Non-randomised men & 8801 & 29067 & $1166(13.2)$ & 2238 (2075 to 2401) \\
\hline Overall total & 47505 & 165513 & $5949(12.5)$ & 2112 (2041 to 2184 ) \\
\hline
\end{tabular}

*Age standardised per 100000 in strata of one year to age structure of Western Australian male population aged 65-83 years. 


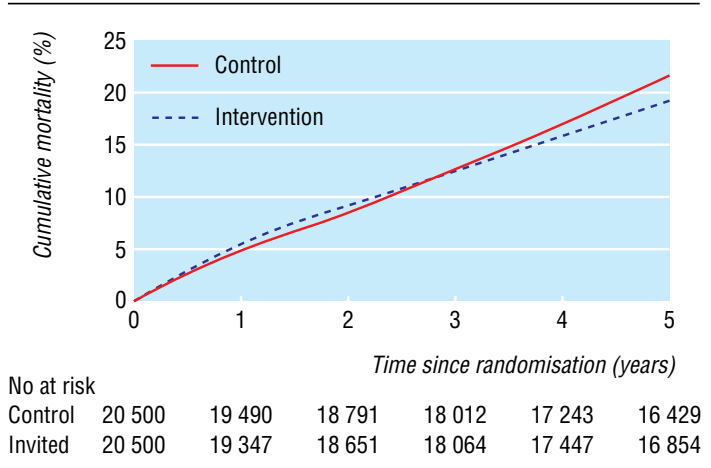

Fig 5 Cumulative all cause mortality from date of randomisation

were not randomised into the multicentre study (through being too old, too ill, or resident in a nursing home). The exclusion of such men optimises the effectiveness of screening by increasing the level of participation. It also removes a group with a relatively high incidence of ruptured abdominal aortic aneurysm. When we applied the criteria for eligibility used in the multicentre study to our study, the response fraction is about $70 \%$. Although attendance fell with increasing age,${ }^{17}$ the lower response seen in our trial was not simply due to inclusion of older men; attendance in men aged $65-74$ years was $74 \%$ compared with the $80 \%$ achieved in the multicentre study. ${ }^{15}$ It is possible that men are more likely to participate if they are invited by their general practitioner rather than a research group.

The mortality from abdominal aortic aneurysm was high in the group of men who were invited but did not attend for screening (table 2). The multicentre study investigators were able to minimise the size of this group by exclusion before randomisation. Strict criteria for considering an individual as "ineligible for screening" may be easier for a general practitioner to apply in the setting of a randomised controlled trial than in real life. A proportion of patients with "other serious health problems" (an exclusion criterion in the multicentre study) will die from ruptured abdominal aortic aneurysm and may have benefited from elective surgery-especially if stenting proves to be more suitable than open repair in high risk cases.

\section{Low mortality from abdominal aortic aneurysm in the control group}

Although our sample size calculation was based on published rates of mortality in Western Australia, ${ }^{3}$ mortality from abdominal aortic aneurysm in our control group was lower than expected (37 $v 55$ deaths). It was also lower than that seen in the multicentre study. The crude cumulative all cause mortality in the control group at four years was similar (12\%) in both studies. In contrast, the crude cumulative mortality from abdominal aortic aneurysm in the control group was $0.33 \%$ in the multicentre study and, despite inclusion of older men, only $0.11 \%$ in our study. Contamination of our control group through ad hoc screening during the trial period may have resulted in the diagnosis and successful treatment of some abdominal aortic aneurysm in this group. More notably, however, linkage to hospital morbidity records showed that a surprisingly large proportion $(1 \%)$ of all randomised men had already had elective surgery for an abdominal aortic aneurysm (table 1). This is substantially greater than in the multicentre study, where the prevalence of survivors of surgery for abdominal aortic aneurysm seemed to be $0.26 \%$. The high frequency of previous surgery in Western Australia may be due to a higher incidence of abdominal aortic aneurysm or greater rates of diagnosis and treatment before screening.

\section{What is already known on this topic}

A screening programme for 65-74 year old men identified as eligible by their general practitioners reduces mortality from abdominal aortic aneurysm.

Such a screening programme is likely to be cost effective.

What this study adds

A screening programme for all 65-83 year old men does not reduce mortality from abdominal aortic aneurysm.

Men aged 65-74 years may benefit from screening provided there are no deaths between recruitment and actual screening

A high background rate of diagnosis and successful treatment may reduce the magnitude of benefit

In men aged $65-83$ years less than $1 \%$ of all deaths are due to abdominal aortic aneurysm

While the overall prevalence of any aneurysm (aortic diameter $\geq 30 \mathrm{~mm}$ ) was higher in our study than in the multicentre study $(7.2 \% v 4.9 \%)$, the proportion of the largest aortas ( $\geq 55 \mathrm{~mm}$ in diameter) in Western Australia was almost half that seen in the multicentre study $(7 \% v 12 \%)$. As the prevalence of large aortas increases with age, this difference is even more remarkable considering the greater age of our cohort. We can only speculate about the reasons for these differences. One possibility is the longstanding and easy availability of imaging (ultrasound and computed tomography) to general practitioners in Australia. This may have resulted in a relatively high level of incidental detection and treatment of large aortas over the past two decades. This aspect of a healthcare system needs to be taken into account when screening for abdominal aortic aneurysm is considered.

\section{Deaths between randomisation and screening}

In our trial, all men were randomised at the beginning of the screening period and inevitably there were deaths from abdominal aortic aneurysm between randomisation and the date of screening. These deaths were not included in our primary analysis and they did not occur in the multicentre study as randomisation was undertaken as the trial progressed. In an ideal world there should be no delay between becoming eligible for screening and actual screening, although in practice this may be a problem.

The chief reasons for our overall result seem to be our failure to identify and exclude men who were unlikely to attend and may have been ineligible for surgery had they attended and a high background level of detection and treatment of abdominal aortic aneurysms in the study community. The success of screening will depend on choosing the best target age group (probably men aged 65-74 years), excluding ineligible men, and minimising delay between becoming eligible for screening and actual screening. It is also important to assess the current rate of elective surgery as in some communities this may already approach a level that reduces the potential benefit of population based screening.

We thank all the men who participated and all the partners who encouraged them to participate. We also thank the State Electoral Commission, the Australian Bureau of Statistics, the registrar general of births, deaths, and marriages, the Health Department of Western Australia, the 
participants' general practitioners, and the hospitals in Perth for providing space in which to conduct screening. Other people associated with the programme were Yvonne Allen, Ann Blakemore, Michelle England, Lorili Jacobs, Gill Kaye, Janet Mitchell, Carol Pearce, Lisa Rich, Lyn Schofield, Jan Sleith, Teresa Warner, and Raylene Williamson. We thank the multicentre aneurysm screening study investigators for their help, especially Alan Scott for advice and encouragement and Theresa Marteau and Jane Colehan, who facilitated use of their quality of life instrument. A final special thanks and dedication goes to Bill Castleden.

Contributors: PEN, KJ, and MML-B were jointly responsible for the conception and design of the study. RWP designed and established the database. MTQL managed the database and was responsible for data linkage and statistical analyses. RJT coordinated the trial. CAS helped coordinate the trial and collected and analysed various aspects of the data. PEN, KJ, MML-B, and JAD interpreted the results and wrote the paper. PEN is the guarantor.

Funding: National Health and Medical Research Council and the National Heart Foundation of Australia.

Competing interests: None declared.

Ethical approval: The Committee for Human Rights at the University of Western Australia approved the protocol for the study.

1 Semmens J, Lawrence-Brown M, Norman P, Codde J, Holman C. The quality of surgical care project: benchmark standards of open resection for abdominal aortic aneurysm in Western Australia. Aust N Z J Surg 1998;68:404-10.

2 Fowkes FGR, Macintyre CCA, Ruckley CV. Increasing incidence of aortic aneurysms in England and Wales. BMJ 1989;298:33-5.

3 Semmens JB, Norman PE, Lawrence-Brown MMD, Bass AJ, Holman CDJ. The incidence of abdominal aortic aneurysm repair in Western Australia for 1985-94: a population-based record linkage study. Br J Surg 1998;87:191-4

4 Reitsma JB, Pleumeekers HJCM, Hoes AW, Kleijnen J, de Groot RM, Jacobs MJ, et al.

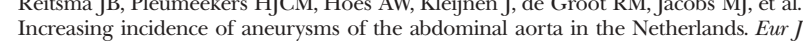
Vasc Endovasc Surg 1996;12:446-51.

5 Scott RAP, Ashton HA, Kay DN. Abdominal aortic aneurysm in 4237 screened patients: prevalence development and management over 6 years. $\mathrm{Br} J$ Surg 1991;78:1122-5.

6 Collin J, Araujo L, Walton J, Lindsell D. Oxford screening programme for abdominal aortic aneurysm in men aged 65 to 74 years. Lancet 1988;ii:613-5.

7 Lederle FA, Johnson GR, Wilson SE, Chute EP, Littooy FN, Bandyk D, et al. Prevalence and associations of abdominal aortic aneurysm detected through screening. Ann Intern Med 1997;126:441-9.

8 Harris P. Reducing the mortality from abdominal aortic aneurysms: need for a national Harris P. Reducing the mortality from abdoming

9 Fowkes F, Ruckley C, Powell J, Greenhalgh R. Screening for abdominal aortic aneurysms. BMJ 1992;305:1013.
10 Cheatle TR. The case against a national screening programme for aortic aneurysms. Ann R Coll Surg Engl 1997;79:90-5.

11 Scott RAP, Wilson NM, Ashton HA, Kay DN. Influence of screening on the incidence of ruptured abdominal aortic aneurysm: 5-year results of a randomised controlled study. Br J Surg 1995;82:1066-70.

12 Wilmink T, Quick C, Hybbard C, Day N. The influence of screening on the incidence of ruptured abdominal aortic aneurysms.J Vasc Surg 1999;30:203-8.

13 Heather B, Poskitt K, Earnshaw J, Whyman M, Shaw E. Population screening reduces mortality rate from aortic aneurysm in men. BrJ Surg 2000;87:750-3.

14 Lindholt J, Juul S, Fasting H, Henneberg E. Hospital costs and benefits of screening for abdominal aortic aneurysms. Results from a randomised population screening trial. Eur I Vasc Endovas Surg 2002;23:55-60.

15 Multicentre Aneurysm Screening Study Group. The multicentre aneurysm screening study into the effect of abdominal aortic aneurysm screening on mortality in men: a randomised controlled trial. Lancet 2002;360:1531-39.

16 Multicentre Aneurysm Screening Study Group. Multicentre aneurysm screening study: cost effectiveness analysis of screening for abdominal aortic aneurysms based on four year results from randomised controlled trial. BMJ 2002;325:1135-8.

17 Jamrozik K, Norman PE, Spencer CA, Parsons RW, Tuohy R, Lawrence-Brown MM, et al. Screening for abdominal aortic aneurysms: lessons from a population-based study. Med J Aust 2000;173:345-50.

18 Nicholls EA, Norman PE, Lawrence-Brown MD, Goodman MA, Pedersen B. Screening for abdominal aortic aneurysms in Western Australia. Aust N Z J Surg 1992;62:858-61.

19 Norman P, Semmens J, Lawrence-Brown M, Holman C. Long term relative survival after surgery for abdominal aortic aneurysm in Western Australia: population based study. BMJ 1998;317:852-6.

(Accepted 14 September 2004)

doi $10.1136 /$ bmj. 38272.478438 .55

School of Surgery and Pathology, University of Western Australia, Fremantle Hospital, PO Box 480, Fremantle, WA 6959, Australia

Paul E Norman associate professor

School of Population Health, University of Queensland, Herston, QLD 4006, Australia

Konrad Jamrozik professor of epidemiology

Mount Medical Centre, Perth, WA 6005, Australia

Michael M Lawrence-Brown vascular surgeon

School of Population Health, University of Western Australia, Crawley, WA 6009, Australia

Max T Q Le research officer

Carole A Spencer research officer

Raywin J Tuohy research assistant

Richard W Parsons statis

Department of Family Medicine, University of Calgary, AB, Canada T2N 1M7

James A Dickinson professor of family medicine

Correspondence to: P Norman pnorman@cyllene.uwa.edu.au 